ARTICLE

Received 4 Apr 2013 | Accepted 29 Oct 2013 | Published 26 Nov $2013 \quad$ DOl: 10.1038/ncomms3841

\title{
Stepwise histone modifications are mediated by multiple enzymes that rapidly associate with nascent DNA during replication
}

\author{
Svetlana Petruk ${ }^{1}$, Kathryn L. Black ${ }^{1}$, Sina K. Kovermann², Hugh W. Brock ${ }^{2} \&$ Alexander Mazo ${ }^{1}$
}

The mechanism of epigenetic inheritance following DNA replication may involve dissociation of chromosomal proteins from parental DNA and reassembly on daughter strands in a specific order. Here we investigated the behaviour of different types of chromosomal proteins using newly developed methods that allow assessment of the assembly of proteins during DNA replication. Unexpectedly, most chromatin-modifying proteins tested, including methylases, demethylases, acetyltransferases and a deacetylase, are found in close proximity to PCNA or associate with short nascent DNA. Histone modifications occur in a temporal order following DNA replication, mediated by complex activities of different enzymes. In contrast, components of several major nucleosome-remodelling complexes are dissociated from parental DNA, and are later recruited to nascent DNA following replication. Epigenetic inheritance of gene expression patterns may require many aspects of chromatin structure to remain in close proximity to the replication complex followed by reassembly on nascent DNA shortly after replication.

\footnotetext{
${ }^{1}$ Department of Biochemistry and Molecular Biology and Kimmel Cancer Center, Thomas Jefferson University, 1020 Locust Street, Philadelphia, Pennsylvania 19107, USA. ${ }^{2}$ Department of Zoology, University of British Columbia, 6270 University Boulevard, Vancouver, British Columbia, Canada V6T 1Z4.

Correspondence and requests for materials should be addressed to A.M. (email: Alexander.Mazo@jefferson.edu).
} 
C urrently, it is not known how the status of gene expression is propagated through $\mathrm{S}$ and $\mathrm{M}$ phases when major changes to chromatin architecture occur. Re-establishment of parental chromatin structure on nascent DNA of future daughter cells in $S$ phase may occur by a two-step mechanism in which most proteins and nucleosomes dissociate from $\mathrm{DNA}^{1}$, but a few molecules can quickly re-associate with DNA following replication. Such proteins or modifications may have an epigenetic role if they mark specific regulatory DNA sequences and then trigger association of the rest of the components of chromatin, probably in a specific order. This would lead to reestablishment of chromatin environment in the regulatory regions of the genes, and allow reconstitution of the gene expression status in daughter cell ${ }^{2,3}$.

Until recently, this model of chromatin assembly remained untested in vivo, because there were no experimental approaches to examine the behaviour of chromosomal proteins other than histones at the time of, and shortly after the passage of the DNA replication complex in vivo. We recently developed several in vivo approaches to examine the proximity of chromosomal proteins to PCNA during replication and at the time of their recruitment to nascent DNA following replication ${ }^{4}$. Using these new tools in Drosophila embryos, we found that TrxG and PcG proteins TRX and $\mathrm{E}(\mathrm{z}), \mathrm{H} 3 \mathrm{~K} 4$ and $\mathrm{H} 3 \mathrm{~K} 27$ histone-methyltrasferases (HMTs), respectively, and PC, a component of the PRC1 complex, associate with their response elements (TREs and PREs) during DNA replication in vivo ${ }^{4}$. These results are in agreement with a previous study showing that several components of the PRC1 complex are stably bound to DNA in the in vitro replication assays ${ }^{5,6}$, and that these proteins are associated in vivo with relatively short stretches of nascent $\mathrm{DNA}^{5}$. Surprisingly, $\mathrm{H} 3 \mathrm{~K} 4 \mathrm{me} 3$ and $\mathrm{H} 3 \mathrm{~K} 27 \mathrm{me} 3$ are not detected in proximity to PCNA or nascent daughter strands of DNA, and initial accumulation of these methyl marks on $\mathrm{H} 3$ was detected only following $S$ phase ${ }^{4}$.

Together, these studies suggest that some TrxG and PcG proteins may function to re-establish active or repressing chromatin environments due to their ability to remain in close proximity to PCNA, or be rapidly recruited to nascent DNA following replication. These findings raise three essential questions: First, can other chromosomal proteins remain in proximity to PCNA or on nascent DNA following replication? Second, why are some histone modifications delayed despite the early presence of histone-modifying enzymes on nascent DNA? Third, what is the order of recruitment of chromosomal proteins to daughter DNA after replication? These issues are particularly important for proteins that are involved in changing the structure of chromatin, some of which are characterized genetically as members of the TrxG and PcG of epigenetic regulators ${ }^{7,8}$, but also include other chromatin modifiers or nucleosome remodelers.

In this study we show that most chromatin-modifying enzymes, but not subunits of chromatin-remodelling complexes, are found in close proximity to PCNA and nascent DNA shortly after DNA replication. We conclude that epigenetic inheritance of gene expression patterns requires that multiple molecules remain in close proximity to the replication complex.

\section{Results}

Examining post-replicative protein assembly by PLA and CAA. To address these questions, we used two assays to survey the behaviour of several groups of histone-modifying and nucleosome remodelling proteins during DNA replication in Drosophila embryos. To examine whether tested proteins are in close proximity to PCNA in the DNA replication complex or bound to DNA following replication we used the Proximity Ligation Assay
(PLA, Olink, Bioscience). The results of PLA were found previously to be in excellent correlation with physical association of TRX, E $(\mathrm{z})$ and PC, with the same DNA fragments as PCNA as detected in sequential re-ChIP assays ${ }^{4}$. Extensive characterization of these re-ChIP assays revealed that these proteins bind to their specific binding DNA sites, TREs and PREs, during or immediately following DNA replication ${ }^{4}$, validating the PLA assays with PCNA as a reliable source of information not only for transient presence of these proteins in close proximity to the DNA replication complex but also for their association or close proximity to DNA during the passage of the replication complex. It should be noted that PLA is a very sensitive technique with a dynamic range that detects proximity of single molecules (Olink), and thus can detect very rare and transient interactions. For comparison, TRX and PC interactions with PCNA are easily detectable by $\mathrm{PLA}^{4}$, but we were not able to detect these interactions in co-immunoprecipitation experiments (Supplementary Fig. S1).

We also used a recently developed Chromatin Assembly Assay (CAA) that is based on labelling of nascent DNA with EdU that is subsequently chemically conjugated with biotin. The antibodies to the tested protein and biotin are then used in a PLA assay to examine proximity of this protein to nascent $\mathrm{DNA}^{4}$. The shortest time for reliable EdU labelling in Drosophila embryos is 4-5 min. With an average speed of DNA replication of about 40 bp per sec, and considering that EdU entering into the nucleus may require some time, 4-5 min of EdU labelling will detect less than $10 \mathrm{~kb}$ of nascent DNA. Previously, we showed that the results of CAA correlate completely with the results of re-ChIP and PLA assays with $\mathrm{PCNA}^{4}$. The results of this and our previous study show that all proteins that are in close proximity to PCNA are also detected in the CAA assays after $4-5 \mathrm{~min}$ of EdU labelling. Proteins that are not detected in these two assays are always detected after longer times of EdU labelling. Thus, simultaneous use of two complementary assays, PLA and CAA, provide a means to survey for proximity to PCNA and time of recruitment of many proteins to nascent DNA at many genomic locations. Re-ChIP assays are not practical for testing multiple proteins because they require prior identification of binding sites at specific targets and large amounts of well-characterized specific antibodies that are not always available from commercial or other sources.

Association of ASH1 and SET1 HMTs with replicating DNA. The HMTs E(z) and TRX are in close proximity to PCNA and nascent $\mathrm{DNA}^{4} . \mathrm{E}(\mathrm{z})$ is the only H3K27 HMT in Drosophila. There are, however, several other H3K4 HMTs in addition to TRX, including ASH1 and dSET1. These proteins belong to different families of HMTs, and have different contributions to the overall levels of methylated H3K4 forms in different tissues ${ }^{9,10}$. Similar to TRX, dSET1 is in close proximity to PCNA (Fig. 1a; all the results on proximity of different proteins to PCNA and nascent DNA are summarized in Table 1). In CAA experiments, dSET1 is in proximity to nascent DNA labelled with EdU for $5 \mathrm{~min}$. Its amount is slightly increased from $10 \mathrm{~min}$ to $2 \mathrm{~h}$ of EdU incorporation. Contrary to TRX and dSET1, ASH1 is not found in proximity to PCNA in the PLA assay (Fig. 1b). Consistent with this, ASH1 is not detected by CAA on nascent DNA until $1 \mathrm{~h}$ labelling with EdU, and its amount is slightly increased at $2 \mathrm{~h}$ of EdU incorporation. The occasional PLA signals that are seen with ASH1 in columns 1-3 (and in some figures below) do not overlap with PCNA- and EdU-labelled nuclei, and represent a typical low antibody background in the control experiments when only one of the tested antibodies is used for the PLA reaction ${ }^{4}$. In the gastrulating Drosophila embryos shown in this figure, the G1 phase is not detectable, $\mathrm{S}$ phase is about $50 \mathrm{~min}$, and most 


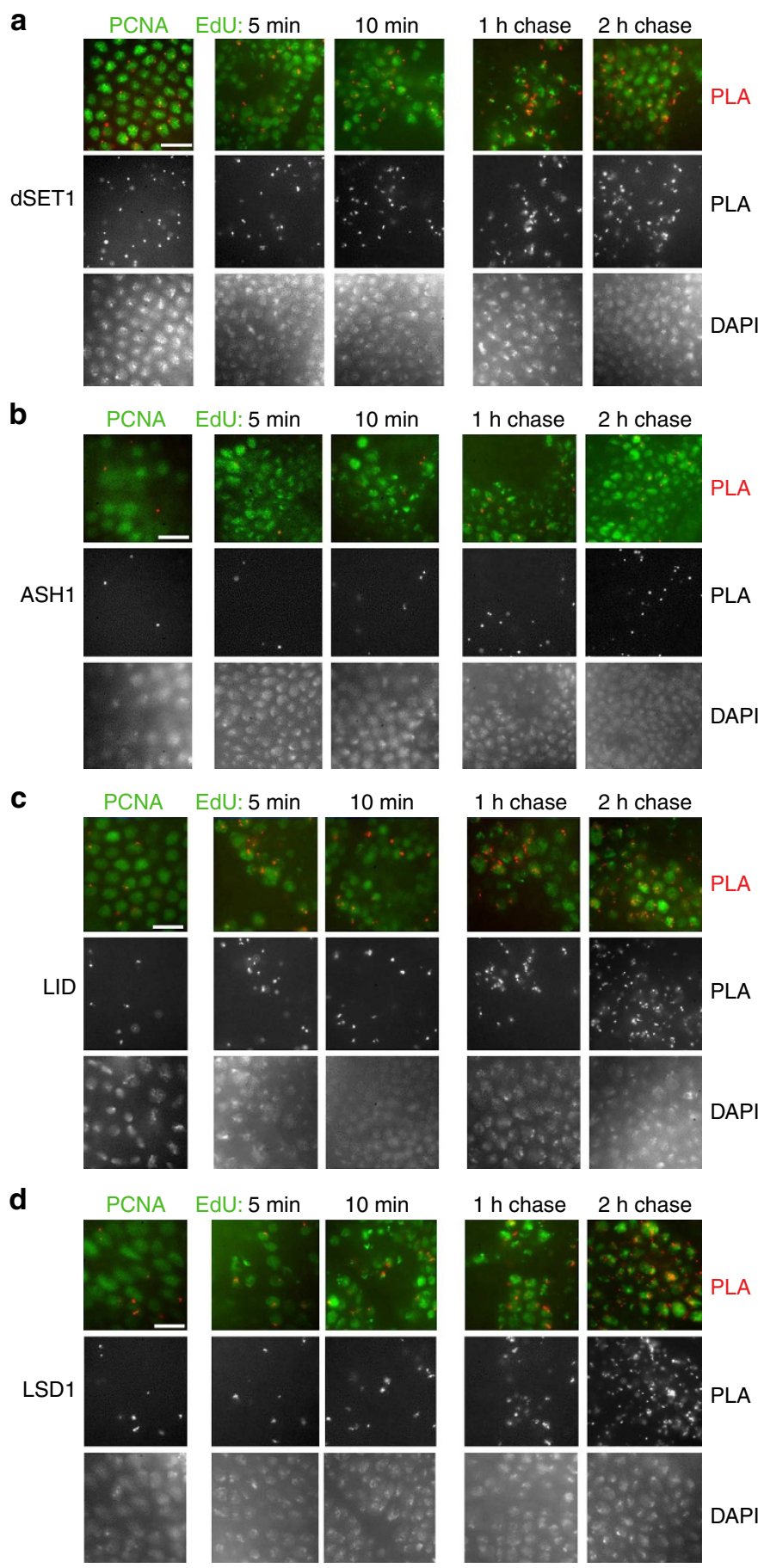

Figure 1 | Proximity of H3K4 HMTs and de-HMTs to PCNA and nascent DNA in Drosophila embryos. (a-d) Proximity of H3K4 HMTS dSET1 (a) and ASH1 (b), and proximity of H3K4 de-HMTs LID (c) and LSD1 (d) to PCNA (left columns) and nascent DNA (columns 2-5) in gastrulating Drosophila embryos. Columns 2, 3, PLA with DNA labelled with EdU for 5 and $10 \mathrm{~min}$. Columns 4, 5, DNA was pulse labelled with EdU for $10 \mathrm{~min}$ and chased for 1 and $2 \mathrm{~h}$. PLA signals are in red. PLA signals from the same images are shown in the middle panels. DAPI staining of DNA of the same images are shown in the bottom rows. The results are from three independent experiments. Scale bar, $10 \mu \mathrm{m}$.

transcription occurs in a G2 phase of variable length that depends on cell type ${ }^{11}$. Thus, ASH1 may be recruited either during mid-S phase or transcriptional G2 interphase. These results suggest that ASH1 may not be required for maturation of chromatin immediately after replication, potentially because it has cell cycle stage-specific activity.

The presence of TRX and dSET1 on nascent DNA in the absence of $\mathrm{H} 3 \mathrm{~K} 4 \mathrm{me} 3$ (ref. 4) suggests that the H3K4 tri-methylation activity of these HMTs is delayed following replication. As these enzymes are also mono- and di-methylate H3K4 to different extents ${ }^{10}$, it is possible that $\mathrm{H} 3 \mathrm{~K} 4 \mathrm{me} 1$ and $\mathrm{H} 3 \mathrm{~K} 4 \mathrm{me} 2$ appear earlier than H3K4me3 after DNA replication. However, similar to H3K4me3 (ref. 4), $\mathrm{H} 3 \mathrm{~K} 4 \mathrm{me} 2$ and $\mathrm{H} 3 \mathrm{~K} 4 \mathrm{me} 1$ are not detected in the proximity to PCNA and are detected on nascent DNA only at about $1 \mathrm{~h}$ after DNA replication, with the subsequent increase after $2 \mathrm{~h}$ of chase following EdU incorporation (Fig. 2 and Table 1).

UTX and CBP mask the activity of $\mathrm{E}(\mathrm{z})$ following replication. The preceding results suggest that the activity of HMTs may be masked by the stronger activities of the lysine demethylases that are in proximity to nascent DNA immediately after replication and during $\mathrm{S}$ phase. Therefore, we examined the behaviour of two H3K4 demethylases during DNA replication. Drosophila Lid (ortholog of the mammalian Rbp-2/JARID1A and Plu-1/JARID1B) and Lsd1 are demethylases with overlapping H3K4me1/2 and $\mathrm{H} 3 \mathrm{~K} 4 \mathrm{me} 2 / 3$ activities, respectively ${ }^{12-15}$. Both demethylases are found in proximity to PCNA and to nascent DNA labelled with EdU for 5 min (Fig. 1c,d).

The simultaneous presence and presumably stronger activities of demethylases may preclude methylation of H3K4 by HMTs until the relative activities of these antagonistic enzymes change at some time after DNA replication. It is difficult to test this idea for $\mathrm{H} 3 \mathrm{~K} 4$ given the large number of potentially redundant $\mathrm{H} 3 \mathrm{~K} 4$ HMTs and demethylases. However, for H3K27, only one HMT and one demethylase with this specificity are present in Drosophila. We have shown previously that H3K27 HMT E(z) is in proximity to nascent DNA after $5 \mathrm{~min}$ of EdU labelling, but $\mathrm{H} 3 \mathrm{~K} 27 \mathrm{me} 3$ is detected only after $1 \mathrm{~h}$ following labelling ${ }^{4}$. UTX is an $\mathrm{H} 3 \mathrm{~K} 27 \mathrm{me} 2 / 3$ demethylase, an ortholog of the mammalian UTX, UTY and JmjD3 (ref. 16). As UTX is also found in proximity to PCNA and nascent DNA early after DNA replication (Fig. 3a), we tested the possibility that the demethylating activity of UTX may prevent $\mathrm{H} 3 \mathrm{~K} 27 \mathrm{me} 3$ accumulation on nascent DNA at early stages following DNA replication. Inhibition of the UTX activity with the recently described compound GSK J4 (ref. 17) leads to the emergence of a considerable number of PLA signals in the CAA assay between H3K27me3 and nascent DNA labelled for EdU for $15 \mathrm{~min}$, whereas non-treated embryos show no PLA signals (Fig. 3d).

Demethylation by UTX may not be the only mechanism causing a delay in $\mathrm{H} 3 \mathrm{~K} 27$ methylation after DNA replication. Importantly, the Drosophila HAT CBP acetylates histone $\mathrm{H} 3$ at $\mathrm{K} 27$, and thus antagonizes methylation of the same residue by $\mathrm{E}(\mathrm{z})^{18}$. Thus, in addition to demethylation of $\mathrm{K} 27 \mathrm{me} 3$ by UTX, proximity of CBP to DNA during replication may provides an additional mechanism in which acetylation of H3K27 may preclude methylation of $\mathrm{H} 3 \mathrm{~K} 27$ by $\mathrm{E}(\mathrm{z})$. Drosophila CBP is in close proximity to PCNA and nascent DNA labelled from $5 \mathrm{~min}$ to $2 \mathrm{~h}$ (Fig. $3 \mathrm{~b}$ ). CBP associates with TRX in the TAC1 complex ${ }^{19}$, and also forms a complex with $\mathrm{UTX}^{20}$. Both TRX ${ }^{4}$ and UTX (Fig. 3a) are associated with DNA after $5 \mathrm{~min}$ of EdU labelling, suggesting that CBP may be present on nascent DNA in two distinct protein complexes. $\mathrm{H} 3 \mathrm{~K} 27 \mathrm{ac}$ is detectable on nascent DNA at 10 min after DNA replication (Fig. 3c). Inhibition of CBP activity with the C646 inhibitor leads to a significant decrease in $\mathrm{H} 3 \mathrm{~K} 27 \mathrm{ac} 15 \mathrm{~min}$ after DNA replication (Fig. 3g), and as a potential consequence to the emergence of H3K27me3 (Fig. 3f). We also detected a loss of $\mathrm{H} 3 \mathrm{~K} 27 \mathrm{ac}$ in embryos treated with UTX inhibitor (Fig. 3e), which is consistent with the proposed role of 
Table 1 | Proximity of chromosomal proteins and modified histones with PCNA and nascent DNA

\begin{tabular}{|c|c|c|c|c|c|c|}
\hline & \multirow[t]{2}{*}{ Function: } & \multirow[b]{2}{*}{ PCNA } & \multicolumn{4}{|c|}{ EdU } \\
\hline & & & $\mathbf{5}^{\prime}$ & $10^{\prime}$ & $1 \mathrm{~h}$ & $2 \mathrm{~h}$ \\
\hline \multicolumn{7}{|c|}{ Lys HMTs: } \\
\hline TRX & H3K4 & + & + & + & + & ++ \\
\hline ASH1 & H3К 4 & - & - & - & + & + \\
\hline dSet1 & H3K4 & + & + & + & + & ++ \\
\hline$E(z)$ & H3K27 & + & + & + & + & ++ \\
\hline Su(Var)3-9 & H3К9 & - & + & + & + & ++ \\
\hline \multicolumn{7}{|c|}{ Lys de-HMTs: } \\
\hline LID & H3К 4 & + & + & + & ++ & +++ \\
\hline LSD1 & H3K4 & + & + & + & ++ & +++ \\
\hline UTX & H3К27 & + & + & + & + & ++ \\
\hline \multicolumn{7}{|c|}{ Arg HMTs: } \\
\hline DART4 & $\mathrm{H} 3 / 4 \mathrm{R}$ & ++ & ++ & ++ & ++ & +++ \\
\hline DART1 & $\mathrm{H} 3 / 4 \mathrm{R}$ & + & + & + & + & ++ \\
\hline CBP & HAT & + & + & + & + & ++ \\
\hline $\mathrm{pCAF}$ & & ++ & ++ & ++ & ++ & +++ \\
\hline HDAC1 & HDAC & + & + & + & + & ++ \\
\hline PC & Ubiquitination & + & + & + & + & ++ \\
\hline $\mathrm{PHO}$ & DNA binding & + & + & + & + & + \\
\hline HP1 & $\begin{array}{c}\text { Heterochromatin } \\
\text { Chromatin remodeling: }\end{array}$ & ++ & ++ & ++ & ++ & +++ \\
\hline BRM & BAP, PBAP & - & - & + & + & ++ \\
\hline Polybromo & PBAP & - & - & + & + & + \\
\hline OSA & BAP & - & - & - & - & ++ \\
\hline ISWI & NURF, CHRAC, ACF & - & - & + & + & ++ \\
\hline H3K4me1 & & - & - & - & + & ++ \\
\hline H3K4me2 & & - & - & - & + & ++ \\
\hline H3K4me3 & & - & - & - & + & ++ \\
\hline H3K27me3 & & - & - & - & + & ++ \\
\hline H3K9me3 & & - & - & + & + & ++ \\
\hline H4R3me2 & & - & + & + & ++ & ++ \\
\hline H3R17me2 & & - & - & - & + & + \\
\hline H3K27ac & & - & - & + & + & ++ \\
\hline
\end{tabular}

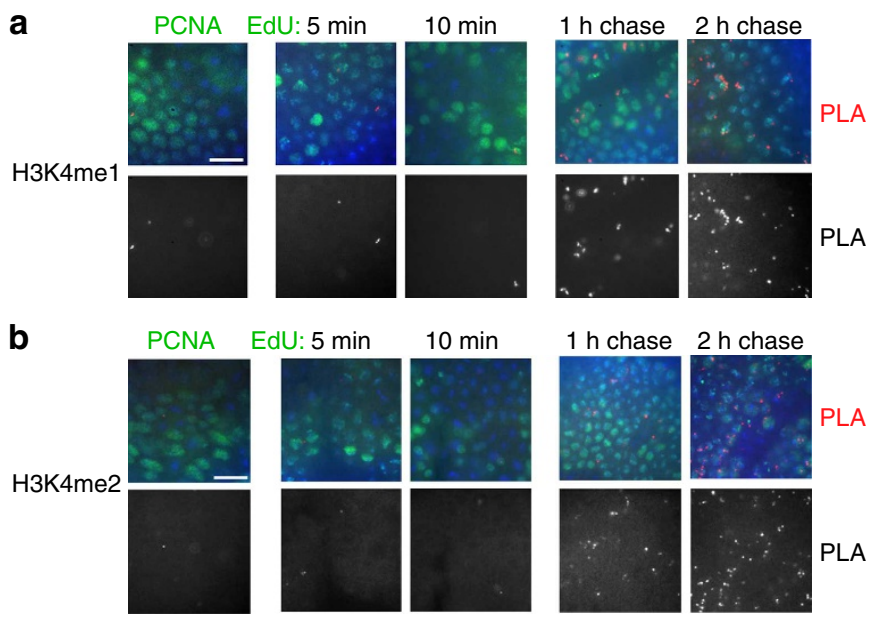

Figure 2 | Proximity of H3K4me1 and H3K4me2 to PCNA and nascent DNA. Antibodies against $\mathrm{H} 3 \mathrm{~K} 4 \mathrm{me} 1$ (a) and $\mathrm{H} 3 \mathrm{~K} 4 \mathrm{me} 2$ (b) were used for PLA with PCNA (left column) and for CAA with EdU-labelled DNA (columns 2-5). Both histone $\mathrm{H} 3$ forms are first detected on DNA labeled with EdU for $1 \mathrm{~h}$. The results are from four independent experiments. Scale bar, $10 \mu \mathrm{m}$.

UTX in CBP activity ${ }^{20}$. In summary, our results indicate that both acetylation of H3K27 by CBP and demethylation of H3K27 by UTX may prevent accumulation of $\mathrm{H} 3 \mathrm{~K} 27 \mathrm{me} 3$ at early stages following DNA replication (scheme in Fig. 3h). It is possible that upregulation of the activity of $\mathrm{E}(\mathrm{z})$ and/or diminishing activities of CBP and UTX contribute to accumulation of $\mathrm{H} 3 \mathrm{~K} 27 \mathrm{me} 3$ at the completion of the $\mathrm{S}$ phase ${ }^{4}$. At this time ( 1 and $2 \mathrm{~h}$ of EdU labelling), the presence of both $\mathrm{H} 3 \mathrm{~K} 27 \mathrm{ac}$ and H3K27me3 (Fig. 3c, Table 1 and (ref. 4)) likely reflects the accumulation of these histone forms in different regions of the genome.

HP1 may be an epigenetic mark of heterochromatin. The combination of PLA and CAA assays also allows us to test the order of recruitment of heterochromatic proteins in the $S$ phase. Methylation of $\mathrm{H} 3 \mathrm{~K} 9$ by $\mathrm{Clr} 4$, a Schizosaccharomyces pombe homologue of the Drosophila Su(Var)3-9, was proposed to create an epigenetic mark that is recognized by the chromo domain of the heterochromatin protein 1 (HP1) that mediates gene silencing ${ }^{21}$, providing a stepwise model for formation of the transcriptionally silent heterochromatin. Another model ${ }^{22}$ suggests that HP1 is recruited to replication forks through direct interaction with histone chaperone CAF1 (ref. 23) that is associated with PCNA. HP1 then recruits SUV39H that induces the full extent of methylation of H3K9. According to this model, H3K9me3 may be transferred to daughter strands, although its role as an epigenetic mark is not clear. We show that HP1 is in proximity to PCNA and with short nascent DNA (Fig. 4a). In contrast, $\mathrm{Su}($ Var)3-9 is not found in proximity to PCNA, but is found on nascent DNA in 5 min after DNA replication (Fig. 4b). $\mathrm{H} 3 \mathrm{~K} 9 \mathrm{me} 3$ is detected shortly after recruitment of this enzyme at $10 \mathrm{~min}$ after DNA replication (Fig. 4c). On the basis of these 
results, we propose a new order of assembly of heterochromatin in Drosophila (Fig. 4d). HP1, but not H3K9me3, may serve as an epigenetic mark during DNA replication. As Su(Var)3-9 and HP1 interact and are interdependent in their localization in Drosophila heterochromatin ${ }^{24}$, it is possible that HP1 recruits $\mathrm{Su}($ Var)3-9 shortly after the passage of the replication fork. a

UTX
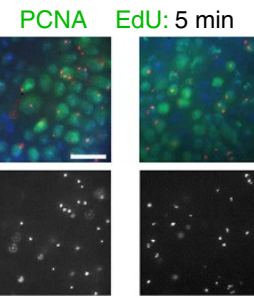

b

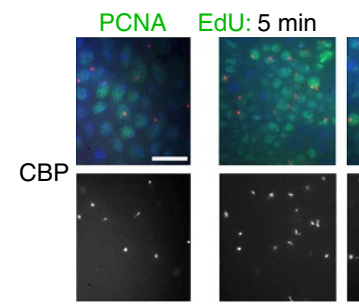

C

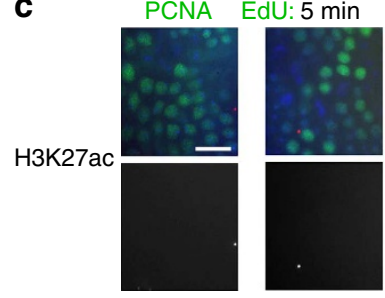

d

EdU: $15 \mathrm{~min}$

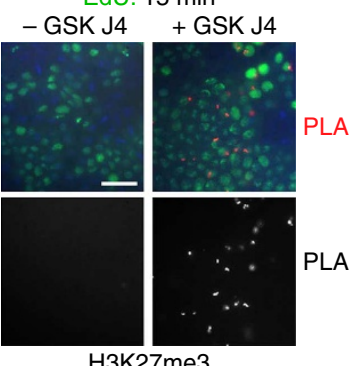

H3K27me3

$\mathbf{f}$

EdU: $15 \mathrm{~min}$
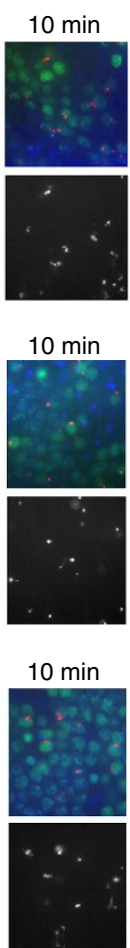
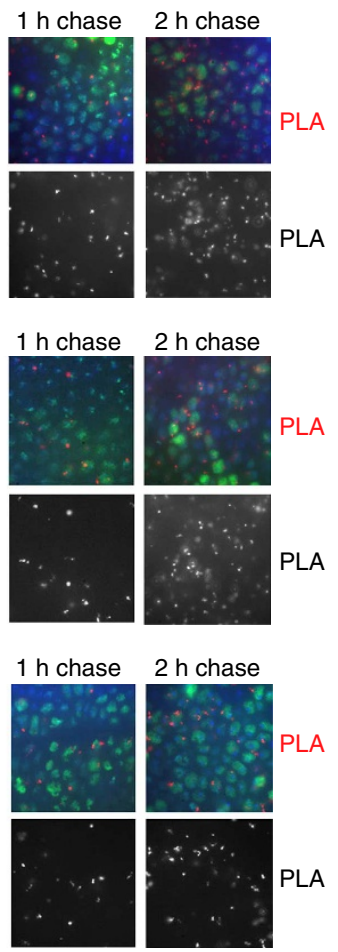

e EdU: $15 \mathrm{~min}$
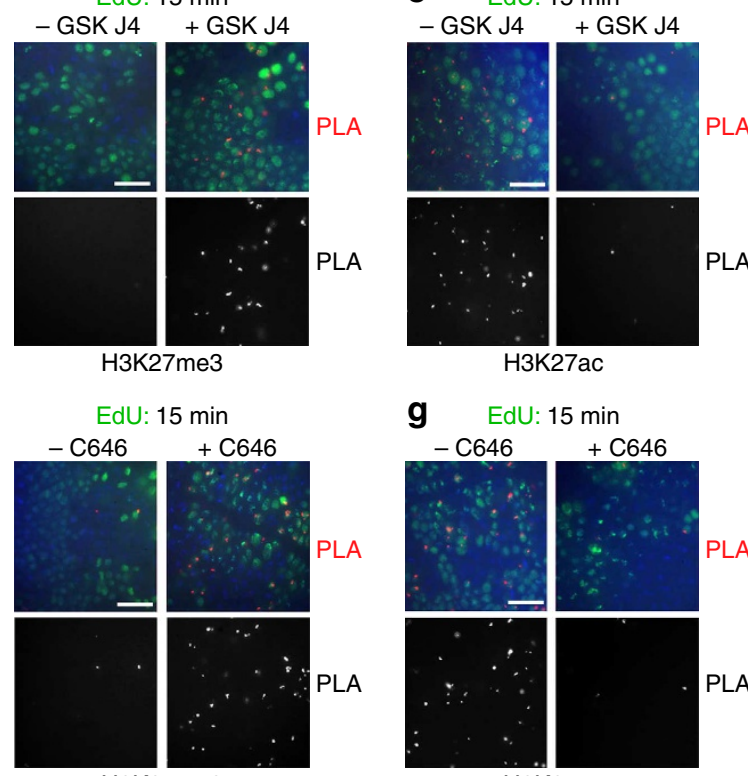

H3K27me3

h DNA replication:

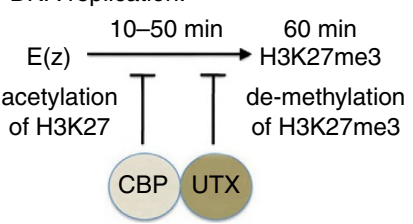

Association of $\mathrm{Su}($ Var)3-9 may lead to fast methylation of $\mathrm{H} 3 \mathrm{~K}$, finalizing the formation of the heterochromatin. Our results do not rule out the possibility that parental $\mathrm{H} 3 \mathrm{~K} 9 \mathrm{me} 3$ is demethylated, transferred to nascent DNA and is methylated at $\mathrm{K} 9$ after $\mathrm{Su}($ Var)3-9 is recruited.

Arginine methyltransferases are in proximity to nascent DNA. To expand our analysis of chromatin proteins that associate with nascent DNA, we examined PRMT arginine methyltransferases that methylate numerous proteins including histones. Although their epigenetic role is not well defined, their ability to methylate histones may potentially impact the epigenetic state of the

a

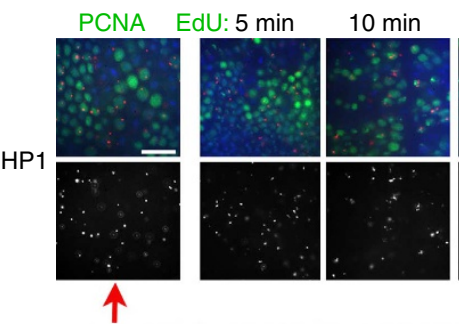

$1 \mathrm{~h}$ chase $2 \mathrm{~h}$ chase

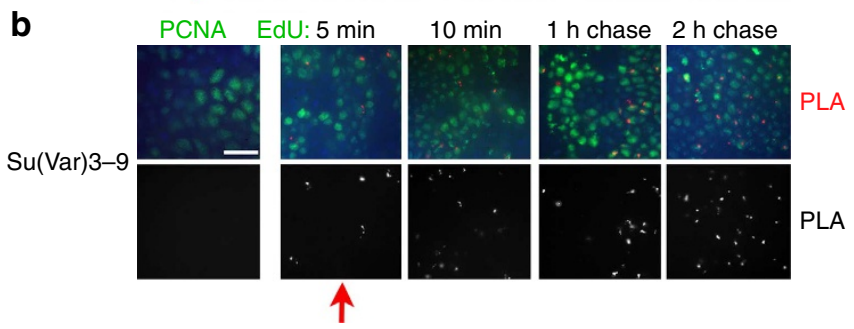

C

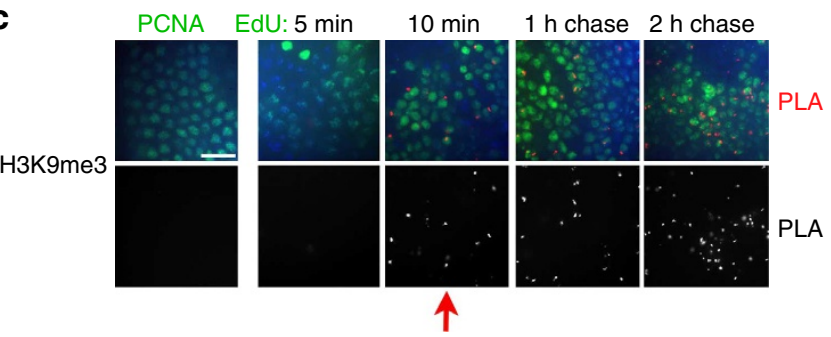

d

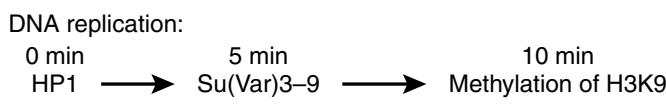

Figure 4 | Heterochromatin proteins are found in proximity to nascent DNA in defined order following replication. Antibodies against Drosophila HP1 protein (a), H3K9 histone methyltransferase Su(Var)3-9 (b) and H3K9me3 (c) were used for PLA with PCNA (left column) and for CAA with EdU-labelled DNA (columns 2-5). Red arrows indicate when proteins are first detected in these experiments. A scheme of proposed sequence of formation of heterochromatin is shown in $\mathbf{d}$. The results are from three independent experiments. Scale bar, $10 \mu \mathrm{m}$.

Figure 3 | Acetylation of H3K27 by CBP and demethylation of H3K27 by UTX may prevent methylation of H3K27 by E(z). (a) Proximity of H3K27 de-HMT UTX, HAT CBP and H3K27ac to PCNA and nascent DNA. (b,c) Inhibition of UTX activity by GSK J4 (b) and CBP activity by C646 (c) leads to early accumulation of H3K27me3 (left) and loss of H3K27ac (right). Embryos were incubated with (left columns) or without (right columns) GSK J4 (b) or C646 (c). They were then labelled with EdU for $15 \mathrm{~min}$, followed by the PLA between biotin and either H3K27me3 or H3K27ac. Following CAA, embryos were immunostained for biotin (EdU, in green). (d) A scheme of proposed inhibition of H3K27 methylation by $\mathrm{E}(\mathrm{z})$ by $\mathrm{CBP}$ and UTX following DNA replication. The results are from four independent experiments. Scale bar, $10 \mu \mathrm{m}$. 
gene $^{25,26}$. Drosophila DART1 and DART4 are arginine methyltransferases (homologous to mammalian PRMT1 and PRMT4/CARM1, respectively) that catalyse formation of the assymetrical dimethylated arginine residues in histones $\mathrm{H} 4$ and H3 (ref. 27). Similar to lysine methyltransferases, DART1 and DART4 are found in proximity to PCNA and nascent DNA labelled with EdU for 5 min (Fig. 5a,b and Table 1). Interestingly, H3R17me2 and H4R3me2, two products of these enzymes, are not associated with PCNA and are detected at nascent DNA at different times following DNA replication (Fig. 5c,d and Table 1). In addition, $\mathrm{pCAF}$, another Drosophila HAT protein, the histone deacetylase HDAC1 and the PcG DNA-binding protein PHO are all in proximity to PCNA and nascent DNA labelled from $5 \mathrm{~min}$ to $2 \mathrm{~h}$ (Figure 6 and Table 1 ). In summary, most tested histone modifiers, chromosomal and DNA-binding proteins are found in close proximity to PCNA and nascent DNA shortly after replication.

Chromatin remodelers are dissociated during DNA replication. Chromatin-remodelling complexes are essential for gene

a

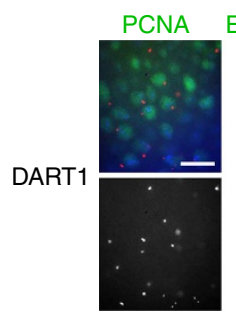

b

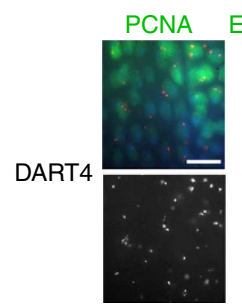

C

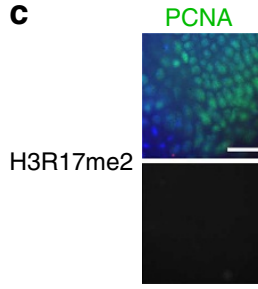

d

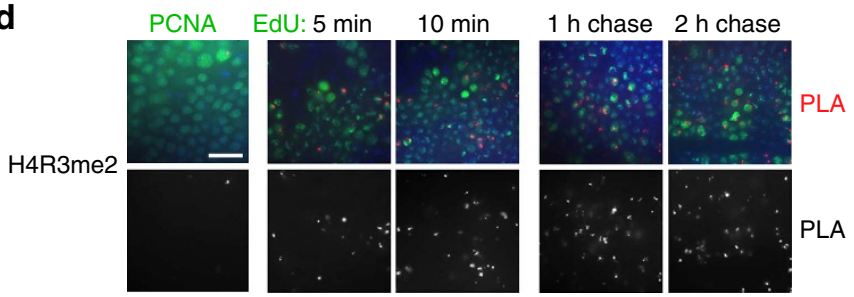

Figure 5 | Proximity of arginine methyltransferases and their products to PCNA and nascent DNA. (a,b) DART1 and DART4 are in proximity to PCNA and nascent DNA. Antibodies against Drosophila DART1 and DART4 were used for PLA with PCNA (left column) and for CAA with EdUlabelled DNA (columns 2-5). (c,d) Histones are methylated at arginines following DNA replication. Antibodies against H3R17me2a and H4R3me2a were used for PLA with PCNA (left column) and for CAA with EdUlabelled DNA (columns 2-5). The results are from three independent experiments. Scale bar, $10 \mu \mathrm{m}$. regulation $^{28,29}$, hence we examined the behaviour of subunits of chromatin-remodelling complexes during DNA replication. ISWI is the catalytic subunit of several distinct ATP-dependent chromatin-remodelling complexes that have essential roles in regulation of higher-order chromatin structure ${ }^{30-32}$. Unlike most of chromatin-modifying enzymes examined above, ISWI is not in proximity to PCNA and appears on nascent DNA only after 10 min of DNA replication (Fig. 7a). The Drosophila TrxG protein BRM is an ATPase ${ }^{33}$ present in two distinct complexes, BAP and PBAP. These complexes share seven identical subunits. The TrxG protein OSA is a component of BAP but not PBAP ${ }^{34}$. In contrast, Polybromo is a component of PBAP but not BAP ${ }^{35}$. Neither BRM nor OSA nor Polybromo are in proximity to PCNA, and they are not found at nascent DNA labelled with EdU for 5 min (Fig. 7b-d). Interestingly, although BRM and Polybromo are first detected on nascent DNA at $10 \mathrm{~min}$, OSA is not detected until $2 \mathrm{~h}$ of EdU incorporation (Fig. $7 \mathrm{~b}-\mathrm{d}$ ). These results suggest that, although both BAP and PBAP complexes dissociate from DNA during replication, these distinct chromatin-remodelling complexes are recruited to their sites on DNA at significantly different times following DNA replication. As the four tested proteins are components of many major chromatin-remodelling complexes (Table 1), our results suggest that chromosomalremodelling complexes are dissociated from DNA for longer periods than most histone-modifying complexes.

Interestingly, a CBP complex with UTX also includes $\mathrm{BRM}^{20}$. Our results suggest that only two components of this complex, UTX and CBP, are in proximity to PCNA and nascent DNA labelled for $5 \mathrm{~min}$ with EdU, whereas BRM is associated with nascent DNA only after 10 min of EdU labelling (Figs 3a,b and 7b). This suggests that the BRM component of the triple UTXCBP-BRM complex is dissociated from this complex during replication. Thus, during replication CBP may exist in two

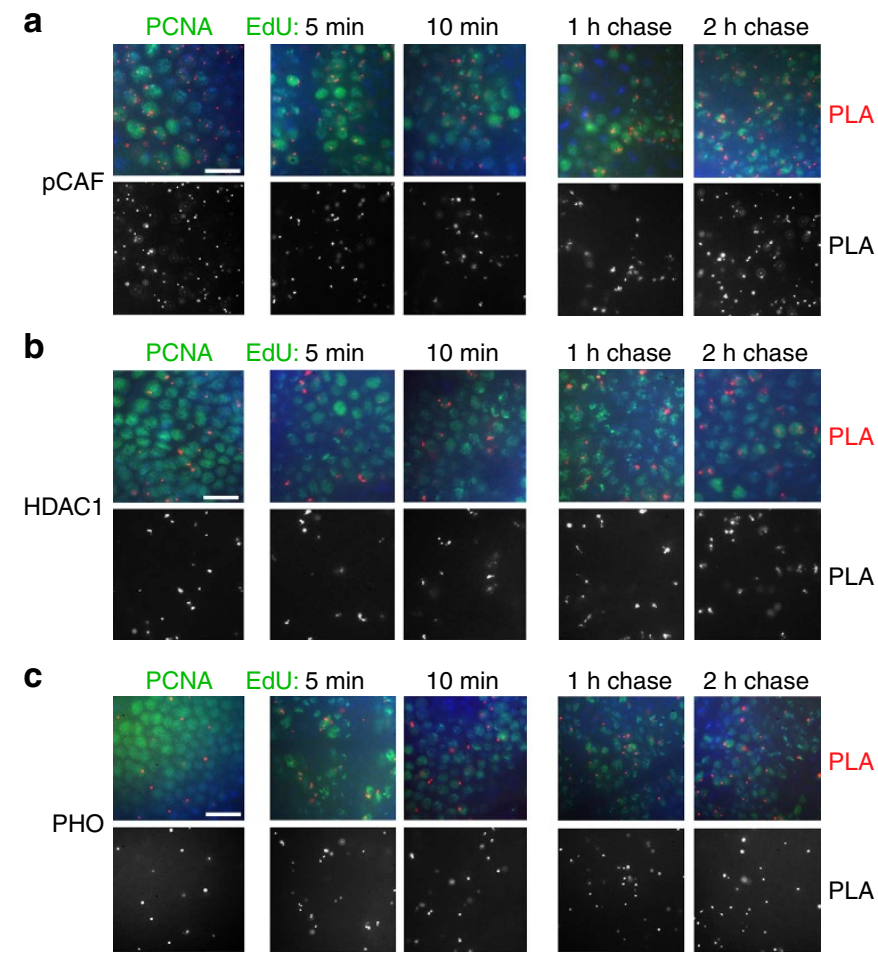

Figure 6 | pCAF and HDAC1 and PHO are in proximity to PCNA and nascent DNA. Antibodies against Drosophila pCAF (a), HDAC1 (b) and PHO (c) were used for PLA with PCNA (left column) and for CAA with EdU-labelled DNA (columns 2-5). The results are from three independent experiments. Scale bar, $10 \mu \mathrm{m}$. 
a
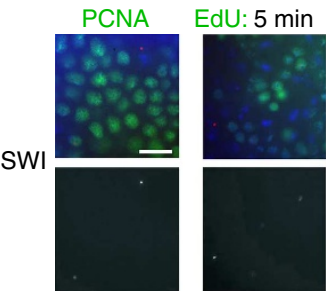

b

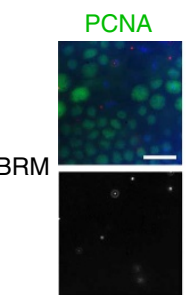

EdU: 5 min

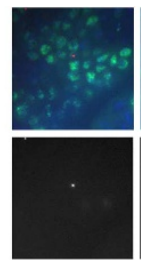

C

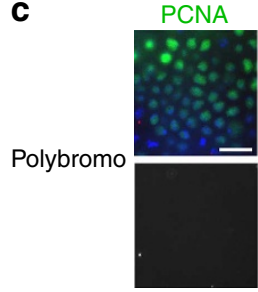

EdU: 5 min

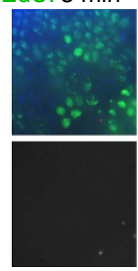

d
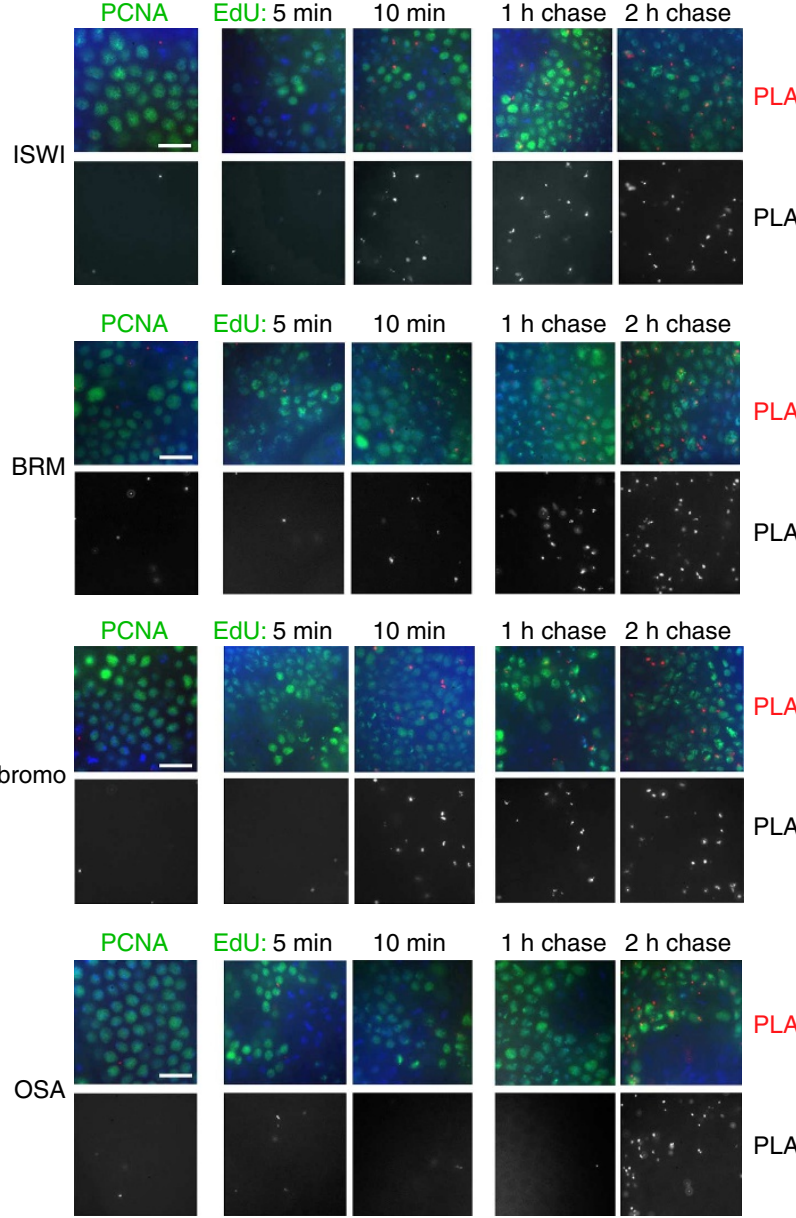

$10 \mathrm{~min}$
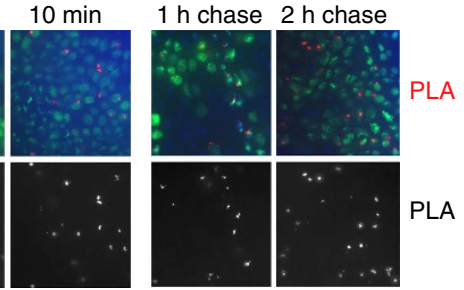

Figure 7 | Recruitment of chromatin remodeling proteins to nascent

DNA. Antibodies against Drosophila chromatin remodelers ISWI (a), BRM (b), Polybromo (c) and OSA (d) were used for PLA with PCNA (left column) and for CAA with EdU-labelled DNA (columns 2-5). ISWI, BRM and Polybromo are first detected on DNA labelled with EdU for $10 \mathrm{~min}$. OSA is first detected on DNA labelled for $2 \mathrm{~h}$. The results are from three independent experiments. Scale bar, $10 \mu \mathrm{m}$.

complexes, with TRX and with UTX, and the BRM component of the UTX-CBP complex is recruited later after DNA replication.

\section{Discussion}

This study examines the recruitment of multiple proteins to daughter DNA following DNA replication in vivo. The surprising finding of this work is that with the exceptions of ASH1 and $\mathrm{Su}(\operatorname{Var}) 3-9$, many histone-modifying proteins that belong to several different functional groups are found in close proximity to PCNA and short nascent DNA. It is likely that these proteins may be transiently dissociated and kept in the vicinity of the replication fork through interactions with PCNA or other replication proteins, and are then rapidly recruited to nascent DNA. It is also possible, that similar to PcG proteins, some of chromatin proteins remain associated with DNA during replication $^{5,6}$. Our results suggest that modifications of histones on daughter DNA occur later after replication, and may be governed by complex activities of histone-modifying proteins associated with nascent DNA. We suggest that histone methylation by itself is unlikely to be an epigenetic mark ${ }^{36}$. Instead, we propose that many chromatin proteins are required early after DNA replication to re-establish transcriptional states.
The methods applied here allow examination of the order of assembly of chromatin. This essential step forward allowed us to examine previously proposed models of epigenetic marking and stepwise assembly of chromatin. In particular, we found that HP1 has an essential early role in formation of heterochromatin. The timing of appearance of other key players suggests that $\mathrm{Su}(\mathrm{Var}) 3-9$ may be recruited by HP1, and that methylation of H3K9 by this protein occurs shortly afterwards. These results agree with previous studies that demonstrated physical interactions between HP1 and Su(Var)3-9. Our studies establish the exact order of events following DNA replication in Drosophila embryos, but do not describe the molecular mechanisms.

This work (Table 1 ) and our previous data ${ }^{4}$ show that unlike histone modifiers, modified histones are accumulated at different times after DNA replication. With the exception of the H4K5ac mark associated with newly synthesized histones, we are unable to determine whether parental, newly synthesized histones or both are acquiring modifications in our experiments. Our data suggest that the histone modification is temporally regulated. Using CAA, it is now possible to functionally assess the roles of histonemodifying enzymes in temporal regulation of histone modification. In particular, we found that methylation of $\mathrm{H} 3$ at $\mathrm{K} 27$ by $\mathrm{E}(\mathrm{z})$, a hallmark of repressed genes in euchromatin, is delayed due to the activities of HAT CBP and de-HMT UTX. The antagonistic effects of these modifiers were described previously 18,20 . Importantly, our work places them in the context of the cell cycle and in the gradual maturation of chromatin following DNA replication.

An important result of this work is that unlike histonemodifying proteins, members of several nucleosome-remodelling complexes dissociate from DNA and re-associate with nascent DNA at different times, perhaps after the end of the $S$ phase, or are developmentally regulated. The mechanisms of recruitment of these proteins following DNA replication are not known. Chromatin remodelers are thought to be recruited to their target genes by transcription factors and modified histones, in particular acetylated nucleosomal histones 37,38 . The results of this and our previous work suggest that modified histones tested so far are detected on nascent DNA with various delays ${ }^{4}$ (Table 1). Thus, one explanation of the late recruitment of chromatin remodelers may stem from delays in accumulation of histone modifications that are essential to recruit these proteins. Significantly, these results suggest that chromatin remodelers do not have direct roles in maintaining epigenetic information during replication and that their roles in transcription may be significantly delayed compared with that of histone-modifying complexes.

Overall, the results of this work demonstrate that in early Drosophila embryos the structure of chromatin may be disrupted for only very short time by DNA replication, and that modification of histones may be a complex orderly process that follows DNA replication. They also suggest that histone modification is unlikely to be an early event in chromatin assembly on nascent DNA or to have a causal role in assembly of specific chromatin environments. We propose that epigenetic marking during replication may require many proteins to be present soon after replication to reconstitute the chromatin environment.

\section{Methods}

PLA assays. Embryos were dechorionated in 50\% bleach and fixed in a 1:1 mixture of $4 \%$ formaldehyde in PBS and heptane for $20 \mathrm{~min}$, washed, blocked and incubated overnight at $4{ }^{\circ} \mathrm{C}$ with primary antibodies from different species. PLA assays (Olink Bioscience) were performed as follows: secondary antibodies with conjugated oligonucleotides (MINUS and PLUS probes) were incubated for $1 \mathrm{~h}$ at $37^{\circ} \mathrm{C}$ (ref. 4). Two oligonucleotides that hybridize to the two PLA probes were added and ligated with DNA ligase for $30 \mathrm{~min}$ at $37^{\circ} \mathrm{C}$. A closed circle forms if proteins are in close proximity. PCR rolling circle amplification by polymerase was 
carried out in the presence of fluorescent-labelled oligonucleotides for $100 \mathrm{~min}$ in the dark at $37^{\circ} \mathrm{C}$. Following PLA reactions, PCNA or EdU were detected in embryos by incubation with FITC-conjugated anti-mouse antibody.

In vivo CAAs. Nascent DNA was labelled with $250 \mu \mathrm{M}$ of EdU in Drosophila Schneider Medium for 5, 10 and a 10 min pulse followed by chase for 1 and $2 \mathrm{~h}$. Embryos were fixed in $4 \%$ formaldehyde in PBS for $20 \mathrm{~min}$. Following incorporation of EdU and fixation, embryos were subjected to Click-iT reaction (Invitrogen) for $30 \mathrm{~min}$ in the dark to add biotin to the EdU. Embryos were washed, blocked in the blocking buffer for antibody incubation (Roche) and incubated with mouse monoclonal anti-biotin antibody and the rabbit antibody of interest at $4{ }^{\circ} \mathrm{C}$ overnight as described ${ }^{4}$. The PLA reaction was performed as described above. Following PLA, EdU was detected by incubation with FITC-conjugated anti-mouse antibody. In experiments using inhibitors, embryos were incubated in the Drosophila Schneider Medium with $40 \mu \mathrm{M}$ of the CBP inhibitor C646 (Sigma) for $1 \mathrm{~h}$ or with $100 \mu \mathrm{M}$ of the UTX inhibitor GSK J4 (Tocris Bioscience) for $1 \mathrm{~h} 30 \mathrm{~min}$ that were dissolved in DMSO. In control experiments, embryos were incubated for the same time with the same concentration of DMSO but without inhibitors. Embryos were then labelled with EdU for $15 \mathrm{~min}$. CAA was performed with antibodies against $\mathrm{H} 3 \mathrm{~K} 4 \mathrm{me} 3$ and $\mathrm{H} 3 \mathrm{~K} 27 \mathrm{ac}$.

Nucleus to nucleus variability in the CAA assays is large. Several reasons may contribute to this variability. Different nuclei in the embryo are at different stages in the $\mathrm{S}$ phase, hence there is variation in the relative amounts of euchromatin and heterochromatin being replicated in particular embryos. It is possible that variability of permeabilization of embryos during 5-10 min labelling with EdU causes some inconsistencies. Embryos are three dimensional, and depending on the $\mathrm{Z}$ plane, different amounts of the peripheral versus central nucleus can be present, thus altering the number of detectable signals.

Antibodies. The following antibodies were used: H3K4mel (1:2,000, Active Motif); H3K4me2 (1:2,000, Active Motif); H3K9me3 (1:2,000, Active Motif); H3R17me2 (1:2,000, Active Motif); H4R3me2 (1:4,000, Active Motif); PCNA (1:5,000, Abcam); ASH1 (ref. 39) (1:1,000); dSET1 (ref. 10) (1:500); LID ${ }^{15}$ $(1: 1,000) ; \operatorname{LSD}^{12}(1: 3,000) ;$ PHO$^{40}(1: 2,000) ; \operatorname{UTX}^{16}(1: 1,000)$, DART1 and DART4 (ref. 27) (1:1,000 and 1:2,000, respectively); CBP and $\operatorname{pCAF}^{19}(1: 2,500$ and $1: 2,000$, respectively); $\operatorname{HDAC1}$ (1:2,000, Abcam); BRM $^{33}$ (1:500); OSA and HP1 (1:3,000 and 1:5,000, respectively, Developmental Hybridoma Bank); ISWI (1:2,000, Abcam); H3K27Ac (1:2,000, Active Motif); Polybromo ${ }^{35}$ (1:2,000); Su(Var)3-9 (1:300, Abcam).

Examination of embryos. Drosophila wild-type strain Oregon R embryos were examined from gastrulation to mid-embryogenesis (4-6h after egg lay, AEL). All antibodies used in this study detect nuclear localization of all examined proteins. The concentrations of all antibodies were adjusted by immunofluorescence to minimize potential non-specific interactions before their use in PLA and CAA assays. Specificity controls included PLA reactions with only one antibody. None of the antibodies used showed any significant number of PLA signals in these control experiments. Specificity of PLA was monitored by examining whether PLA signals are detected only in the PCNA- and EdU-labelled nuclei. PLA signals were counted in 300-400 nuclei from several embryos in at least three independent experiments. Figure images reflect the results of a typical experiment. The assessment of the numbers of PLA signals in Table 1 is explained in the legend to Table 1.

Western blotting. A quantity of $0.25 \mathrm{mg}$ of nuclear extract prepared from Drosophila embryos was immunoprecipitated with PCNA antibody or with IgG as a negative control. The immunoprecipitated material was analysed by western blotting with antibodies against Polycomb and Trithorax.

\section{References}

1. Corpet, A. \& Almouzni, G. Making copies of chromatin: the challenge of nucleosomal organization and epigenetic information. Trends. Cell Biol. 19, 29-41 (2009).

2. Bonasio, R., Tu, S. \& Reinberg, D. Molecular signals of epigenetic states. Science 330, 612-616 (2010).

3. Zhu, B. \& Reinberg, D. Epigenetic inheritance: uncontested? Cell Res. 21, 435-441 (2011).

4. Petruk, S. et al. TrxG and PcG Proteins but not methylated histones remain associated with dna through replication. Cell 150, 922-933 (2012).

5. Francis, N. J., Follmer, N. E., Simon, M. D., Aghia, G. \& Butler, J. D. Polycomb proteins remain bound to chromatin and DNA during DNA replication in vitro. Cell 137, 110-122 (2009).

6. Lengsfeld, B. M., Berry, K. N., Ghosh, S., Takahashi, M. \& Francis, N. J. A Polycomb complex remains bound through DNA replication in the absence of other eukaryotic proteins. Sci. Rep. 2, 661 (2012).

7. Muller, J. \& Kassis, J. A. Polycomb response elements and targeting of Polycomb group proteins in Drosophila. Curr. Opin. Genet. Dev. 16, 476-484 (2006).
8. Ringrose, L. \& Paro, R. Polycomb/Trithorax response elements and epigenetic memory of cell identity. Development 134, 223-232 (2007).

9. Byrd, K. N. \& Shearn, A. ASH1, a Drosophila trithorax group protein, is required for methylation of lysine 4 residues on histone H3. Proc. Natl Acad. Sci. USA 100, 11535-11540 (2003).

10. Ardehali, M. B. et al. Drosophila Set1 is the major histone H3 lysine 4 trimethyltransferase with role in transcription. EMBO J. 30, 2817-2828 (2011).

11. Shermoen, A. W., McCleland, M. L. \& O'Farrell, P. H. Developmental control of late replication and S phase length. Curr. Biol. 20, 2067-2077 (2010).

12. Di Stefano, L., Ji, J. Y., Moon, N. S., Herr, A. \& Dyson, N. Mutation of Drosophila Lsd1 disrupts H3-K4 methylation, resulting in tissue-specific defects during development. Curr. Biol. 17, 808-812 (2007).

13. Di Stefano, L. et al. Functional antagonism between histone H3K4 demethylases in vivo. Genes Dev. 25, 17-28 (2011).

14. Lee, N. et al. The trithorax-group protein Lid is a histone $\mathrm{H} 3$ trimethyl-Lys4 demethylase. Nat. Struct. Mol. Biol. 14, 341-343 (2007).

15. Secombe, J., Li, L., Carlos, L. \& Eisenman, R. N. The trithorax group protein Lid is a trimethyl histone $\mathrm{H} 3 \mathrm{~K} 4$ demethylase required for $\mathrm{dMyc}$-induced cell growth. Genes Dev. 21, 537-551 (2007).

16. Smith, E. R. et al. Drosophila UTX is a histone H3 Lys27 demethylase that colocalizes with the elongating form of RNA polymerase II. Mol. Cell Biol. 28, 1041-1046 (2008).

17. Kruidenier, L. et al. A selective jumonji H3K27 demethylase inhibitor modulates the proinflammatory macrophage response. Nature 488, 404-408 (2012).

18. Tie, F. et al. CBP-mediated acetylation of histone $\mathrm{H} 3$ lysine 27 antagonizes Drosophila Polycomb silencing. Development 136, 3131-3141 (2009).

19. Petruk, S. et al. Trithorax and dCBP acting in a complex to maintain expression of a homeotic gene. Science 294, 1331-1334 (2001).

20. Tie, F., Banerjee, R., Conrad, P. A., Scacheri, P. C. \& Harte, P. J. Histone demethylase UTX and chromatin remodeler BRM bind directly to CBP and modulate acetylation of histone H3 lysine 27. Mol. Cell Biol. 32, 2323-2334 (2012).

21. Bannister, A. J. et al. Selective recognition of methylated lysine 9 on histone $\mathrm{H} 3$ by the HP1 chromo domain. Nature 410, 120-124 (2001).

22. Maison, C. \& Almouzni, G. HP1 and the dynamics of heterochromatin maintenance. Nat. Rev. Mol. Cell Biol. 5, 296-304 (2004)

23. Murzina, N., Verreault, A., Laue, E. \& Stillman, B. Heterochromatin dynamics in mouse cells: interaction between chromatin assembly factor 1 and HP1 proteins. Mol. Cell 4, 529-540 (1999).

24. Schotta, G. et al. Central role of Drosophila SU(VAR)3-9 in histone H3-K9 methylation and heterochromatic gene silencing. EMBO J. 21, 1121-1131 (2002).

25. Balint, B. L. et al. Arginine methylation provides epigenetic transcription memory for retinoid-induced differentiation in myeloid cells. Mol. Cell Biol. 25, 5648-5663 (2005).

26. Bedford, M. T. \& Clarke, S. G. Protein arginine methylation in mammals: who, what, and why. Mol. Cell 33, 1-13 (2009).

27. Boulanger, M. C. et al. Characterization of the Drosophila protein arginine methyltransferases DART1 and DART4. Biochem J. 379, 283-289 (2004).

28. Mellor, J. The dynamics of chromatin remodeling at promoters. Mol. Cell 19 , 147-157 (2005).

29. Fry, C. J. \& Peterson, C. L. Chromatin remodeling enzymes: who's on first? Curr. Biol. 11, R185-R197 (2001).

30. Siriaco, G., Deuring, R., Chioda, M., Becker, P. B. \& Tamkun, J. W. Drosophila ISWI regulates the association of histone $\mathrm{H} 1$ with interphase chromosomes in vivo. Genetics 182, 661-669 (2009).

31. Corona, D. F. et al. ISWI regulates higher-order chromatin structure and histone $\mathrm{H} 1$ assembly in vivo. PLoS Biol. 5, e232 (2007).

32. Sala, A. et al. Genome-wide characterization of chromatin binding and nucleosome spacing activity of the nucleosome remodelling ATPase ISWI. EMBO J. 30, 1766-1777 (2011).

33. Papoulas, O. et al. The Drosophila trithorax group proteins BRM, ASH1 and ASH2 are subunits of distinct protein complexes. Development 125, 3955-3966 (1998).

34. Mohrmann, L. et al. Differential targeting of two distinct SWI/SNF-related Drosophila chromatin-remodeling complexes. Mol. Cell Biol. 24, 3077-3088 (2004).

35. Nakayama, T., Shimojima, T. \& Hirose, S. The PBAP remodeling complex is required for histone $\mathrm{H} 3.3$ replacement at chromatin boundaries and for boundary functions. Development 139, 4582-4590 (2012).

36. Hansen, K. H. et al. A model for transmission of the H3K27me3 epigenetic mark. Nat. Cell Biol. 10, 1291-1300 (2008)

37. Clapier, C. R. \& Cairns, B. R. The biology of chromatin remodeling complexes Annu. Rev. Biochem. 78, 273-304 (2009).

38. Peterson, C. L. \& Logie, C. Recruitment of chromatin remodeling machines. J. Cell Biochem. 78, 179-185 (2000).

39. Rozovskaia, T. et al. Trithorax and ASH1 interact directly and associate with the trithorax group-responsive bxd region of the Ultrabithorax promoter. $\mathrm{Mol}$. Cell Biol. 19, 6441-6447 (1999). 
40. Brown, J. L., Mucci, D., Whiteley, M., Dirksen, M. L. \& Kassis, J. A. The Drosophila Polycomb group gene pleiohomeotic encodes a DNA binding protein with homology to the transcription factor YY1. Mol. Cell 1, 1057-1064 (1998).

\section{Acknowledgements}

We thank A. Shilatifard, T. Kusch, L. Di Stefano, R. Eisenman, S. Richard, J. Tamkun, S. Hirose for antibodies. This work was supported by the following grants: NIH R01GM075141, NIH P01CA129242 to A.M., and a grant from the Canadian Institutes of Health Research to H.W.B.

\section{Author contributions}

S.P., A.M. and H.W.B. designed the study, analysed the data and wrote the paper. S.P. performed experiments shown in Figs $1-3$ and 6 and Supplementary Fig. S1, K.L.B. performed experiments shown in Figs 4,5 and 7. S.K.K. assisted in experiments shown in Fig. 4b-d. All authors discussed the results and commented on the manuscript.

\section{Additional information}

Supplementary Information accompanies this paper at http://www.nature.com/ naturecommunications

Competing financial interests: The authors declare no competing financial interests.

Reprints and permission information is available online at http://npg.nature.com/ reprintsandpermissions/

How to cite this article: Petruk, S. et al. Stepwise histone modifications are mediated by multiple enzymes that rapidly associate with nascent DNA during replication. Nat. Commun. 4:2841 doi: 10.1038/ncomms3841 (2013). 